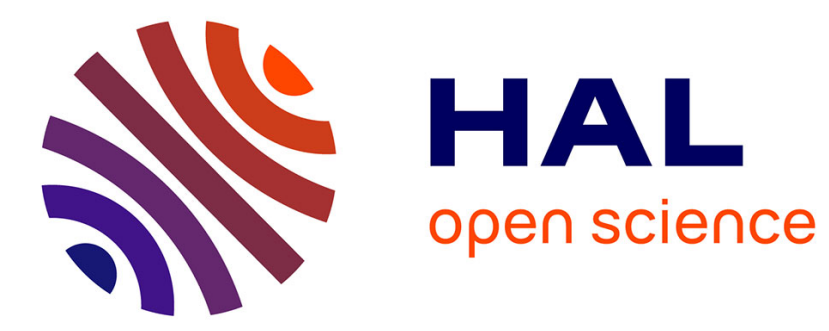

\title{
Ultrasonic Attenuation Measurements in Neutron-Irradiated Silicon
}

\author{
M. Coeck, C. Laermans
}

\section{To cite this version:}

M. Coeck, C. Laermans. Ultrasonic Attenuation Measurements in Neutron-Irradiated Silicon. Journal de Physique IV Proceedings, 1996, 06 (C8), pp.C8-625-C8-628. 10.1051/jp4:19968134 jpa-00254566

\section{HAL Id: jpa-00254566 https://hal.science/jpa-00254566}

Submitted on 1 Jan 1996

HAL is a multi-disciplinary open access archive for the deposit and dissemination of scientific research documents, whether they are published or not. The documents may come from teaching and research institutions in France or abroad, or from public or private research centers.
L'archive ouverte pluridisciplinaire HAL, est destinée au dépôt et à la diffusion de documents scientifiques de niveau recherche, publiés ou non, émanant des établissements d'enseignement et de recherche français ou étrangers, des laboratoires publics ou privés. 


\title{
Ultrasonic Attenuation Measurements in Neutron-Irradiated Silicon
}

\author{
M. Coeck*,** and C. Laermans* \\ * Katholieke Universiteit Leuven, Department of Physics, Celestijnenlaan 200D, 3001 Heverlee, \\ Belgium \\ ** SCK-CEN, Department BR2, Boeretang 200, 2400 Mol, Belgium
}

\begin{abstract}
At low temperatures, amorphous and partly disordered solids exhibit properties which are different from those in crystals. These anomalies can phenomenologically be described by low-energy excitations which are characterized by a broad distribution of energy and relaxation times : the socalled tunneling states (TS). It was first believed that these TS can only occur in typical glass-forming amorphous solids with a low average coordination of the individual atoms, and also in partly disordered, low-coordinated solids such as neutron-irradiated $\mathrm{SiO}_{2}$, TS have been observed. In order to explore the possibility of the presence of TS in topological more constrained disordered solids, ultrasonic attenuation measurements were started on the fourfold coordinated silicon which was irradiated with fast neutrons to create large regions of lattice disorder. In this paper we will present and discuss the first results of these measurements and we will compare them with results of similar measurements on unirradiated, single-crystalline silicon.
\end{abstract}

\section{INTRODUCTION}

The ultrasonic attenuation is one of the many properties of which the behavior at low temperature in crystals differs from that in amorphous or partly disordered materials. According to the tunneling model [1], this anomaly can be explained by the existence of low-energy excitations which are characterized by a broad distribution of energy and relaxation times. These excitations are also known as tunneling states (TS). In spite of the fact that the tunneling model can successfully describe all dynamic properties, it is purely phenomenological and it gives no indication on the microscopic nature of the TS.

In order to contribute to the search for the microscopic origin of these TS, a study of neutron-irradiated quartz is being carried out in our laboratory. 'Glassy' anomalies, induced by irradiation, were found in the partly disordered crystals. The study also revealed an anisotropic behavior of the TS, leading to a description of TS as a rotation of coupled $\mathrm{SiO}_{4}$ tetrahedra [2], which implies that the presence of TS needs to be accompanied by a certain degree of freedom.

It was first believed that TS can only occur in glass-forming amorphous solids with a low average coordination of the individual atoms like $\mathrm{Se}, \mathrm{SiO}_{2}$, and the chalcogenides. Until now it is not quite established that these states can also exist in higher coordinated materials such as amorphous silicon and germanium, which are fourfold coordinated. Amorphous Si can only be prepared in films and 'glassy' anomalies have been reported in certain films $[3,4,5]$, however it is believed that these anomalies are due to voids [5] or lowdensity regions [4]. We started a study of bulk $\mathrm{Si}$ and this paper discusses the behavior of the ultrasonic attenuation in a temperature range from $0.3 \mathrm{~K}$ to $100 \mathrm{~K}$, in pure and partly disordered single-crystalline bulk Si. To induce defects and large regions of displaced atoms in these samples, the Si rod was irradiated with fast neutrons. The purpose is to investigate whether the fast-neutron irradiation can induce the TS in bulk Si single crystals like in irradiated quartz, in spite of the high coordination.

Here we will present the first results of ultrasonic attenuation measurements as a function of temperature on bulk Si which was partly disordered by a fast-neutron dose of $1.7 \times 10^{21} \mathrm{n} / \mathrm{cm}^{2}(\mathrm{E}>0.1 \mathrm{MeV})$. The results 
will be qualitatively analyzed in the framework of the tunneling model and compared to those for unirradiated single-crystalline $\mathrm{Si}$.

\section{THEORETTCAL CONSIDERATIONS}

The basic assumption of the tunneling model is that atoms or groups of atoms can tunnel in a double, asymmetric potential well. The difference between the eigenstates is given by $E=\sqrt{\Delta^{2}+\Delta_{0}^{2}}$, where $\Delta$ represents the asymmetry of the potential well. $\Delta_{0}$ is the tumnel splitting which can be written as $\Delta_{0}=\hbar \Omega \exp (-\lambda)$, where $\lambda$ describes the overlap of the wave functions. $\hbar \Omega / 2$ is the ground state energy of the particle in an isolated well. The tunneling model also assumes that $\Delta$ and $\lambda$ are independent of each other and that they have a uniform distribution : $P(\Delta, \lambda) d \Delta d \lambda=\bar{P} d \Delta d \lambda$, where $\bar{P}$ is a constant and represents the density of states of the TS.

To describe the effect of the TS on the low-temperature ultrasonic properties, two different mechanisms have to be considered : the resonant and the relaxation process. Since the contribution of the resonant absorption is neglectable in these high-energy experiments, we will only discuss the relaxation process. This process results from changes in the occupation of the TS themselves which occur when the sound wave modulates the energy splittings $\Delta$. Relaxation of these TS of which the thermal equilibrium was disturbed, occurs via interaction with the thermal phonons. At low temperatures, the dominant process is the one-phonon process, giving rise to a relaxation rate which can be written as $\tau^{-1}=K_{3}\left(\Delta_{0} / E\right)^{2}(E / 2 k)^{3} \operatorname{coth}(E / 2 k T)$, with $K_{3}=\left(4 k^{3} / \rho \pi \hbar^{4}\right)\left(\gamma_{l}{ }^{2} / v_{l}^{5}+\gamma_{t}{ }^{2} / v_{t}^{5}\right)$, where $\gamma_{l}$ and $\gamma_{t}$ represent the coupling of the TS with respectively longitudinal and transverse waves. The general expression for the attenuation $\alpha$ due to this process can be written as [6]:

$$
\alpha=\frac{\gamma_{l}^{2}}{\rho v_{l}^{3} k T} \int_{E_{\min }}^{E_{\max }} d E \int_{u_{\min }}^{1}\left(\frac{\omega^{2} \tau}{1+\omega^{2} \tau^{2}}\right)\left(\frac{\bar{P}}{u\left(1-u^{2}\right)^{1 / 2}}\right)\left(\frac{\Delta}{E}\right)^{2} \operatorname{sech}^{2}\left(\frac{E}{2 k T}\right) d u
$$

with $u=\Delta_{0} / E$.

Analytical solutions can be derived for the limiting cases $\omega \tau_{\mathrm{m}} \gg>1$ and $\omega \tau_{\mathrm{m}}<<1$, where $\tau_{\mathrm{m}}$ represents the smallest relaxation time of the TS.

At the lowest temperatures, where $\omega \tau_{m} \gg 1$, the ultrasonic attenuation due to the relaxation process is expected to be frequency independent and proportional to $T^{3}$ :

$$
\alpha=\frac{\pi^{3} k^{3} \bar{P} \gamma_{l}^{2}}{24 \rho^{2} \hbar^{4} v_{l}^{3}}\left(\frac{\gamma_{l}^{2}}{v_{l}^{5}}+\frac{2 \gamma_{t}^{2}}{v_{t}^{5}}\right) T^{3}
$$

The $\omega \tau_{\mathrm{m}} \ll 1$ regime holds at higher temperatures and in this case the attenuation can be written as :

$$
\alpha=\frac{\pi \bar{P} \gamma_{l}^{2}}{2 \rho v_{l}^{2}} \omega
$$

This means that at higher temperatures a temperature-independent absorption, a so-called plateau, can be expected which is proportional to the frequency.

\section{SAMPLE PROPERTIES AND EXPERIMENTAL PROCEDURES}

For our experiments two pure single-crystalline Si cylinders were used ( $3 \mathrm{~mm}$ diameter and $15 \mathrm{~mm}$ length). The rod axis of the samples is parallel to the crystallographic [100] axis. One sample, labeled UNIRRSi, remained unirradiated and the other one was irradiated in the BR2 reactor at the SCK·CEN with a fast-neutron dose of $1.7 \times 10^{21} \mathrm{n} / \mathrm{cm}^{2}(\mathrm{E}>0.1 \mathrm{MeV})$. This sample is further indicated as IRRSi.

The mass densities of the samples was measured using a hydrostatic method and this resulted in $2.333 \pm 0.004$ $\mathrm{g} / \mathrm{cm}^{3}$ for the UNIRRSi and $2.327 \pm 0.002 \mathrm{~g} / \mathrm{cm}^{3}$ for the IRRSi sample. 
The behavior of the ultrasonic attenuation is studied as a function of temperature (from 0.3 to $100 \mathrm{~K}$ ), for different frequencies $(140$ and $320 \mathrm{MHz}$ ). The ultrasonic attenuation measurements are based on a standard pulse-echo method. The conversion of the electromagnetic signal into an elastic wave is established using a $\mathrm{LiNbO}_{3}$ transducer which is attached to the samples by means of a very thin layer of DC 200 silicon fluid.

\section{EXPERIMENTAL RESULTS AND DISCUSSION.}

In fig. 1 the measured data for the UNIRRSi and the IRRSi samples are presented. A temperature-independent background attenuation was subtracted.

For the UNIRRSi sample a typical crystalline behavior can be observed: within the experimental accuracy of the measurement no change in ultrasonic attenuation with increasing temperature is measured until $25 \mathrm{~K}$, after which a very steep rise is seen. This rise can be attributed to the anharmonic three-phonon processes which are typical for pure crystals measured at high frequencies. A more detailed discussion of the behavior at high frequencies of the ultrasonic attenuation as a function of temperature for the UNIRRSi sample can be found in [7].

On the other hand, the irradiated sample clearly shows a measurable increase of the ultrasonic attenuation from $1 \mathrm{~K}$ on, followed by a leveling off at about 10 to $15 \mathrm{~K}$, after which again a more steep rise sets in. When the lowest temperature range is studied in detail, it is seen that the increase of the ultrasonic attenuation with temperature can be described by a $T^{3 .}$ This is similar to what is observed in amorphous solids and in irradiated quartz. The attenuation is however very small and at $2 \mathrm{~K}$ it is a factor of about 100 smaller than in vitreous silica [8] or borosilicate glass [9]. In the framework of the tunneling model this behavior corresponds to the $\omega \tau_{\mathrm{m}} \gg>1$ regime of the relaxational interaction of the TS with the phonons (see eq. 2). Preliminary experiments at other frequencies showed that the $T^{3}$-behavior is frequency-independent, which is in agreement with the predictions according to the tunneling model (see eq. 2).

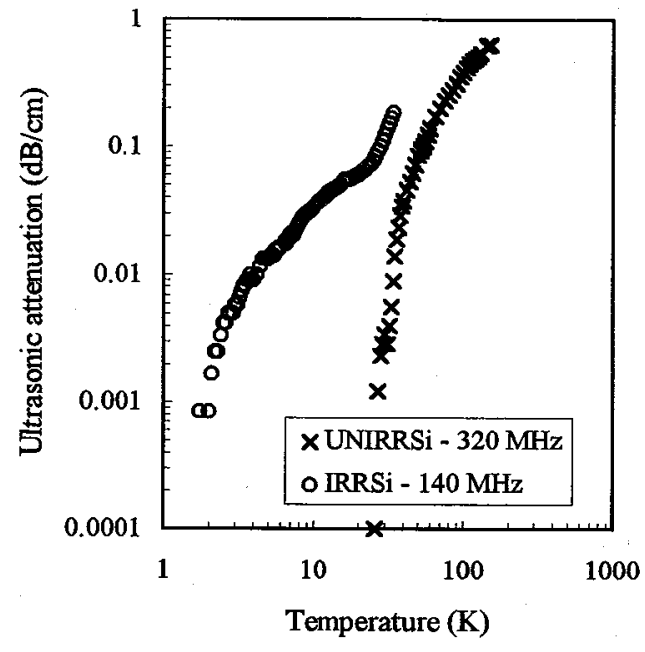

Fig. 1: Ultrasonic attenuation as a function of temperature for pure single-crystalline bulk $\mathrm{Si}$ (UNIRRSi) and for neutron-irradiated Si (IRRSi).

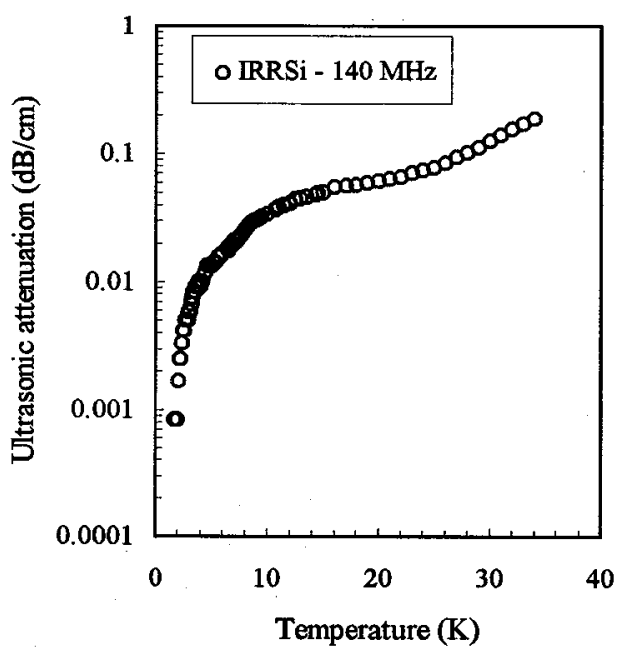

Fig. 2: Linear-logarithmic plot of the ultrasonic attenuation as a function of temperature for neutron-irradiated $\mathrm{Si}$.

In fig. 2 the data are redrawn in a linear-logarithmic plot in order to have a more detailed view of the behavior of the ultrasonic attenuation at the temperature of the shoulder. This figure clearly shows a temperature-independent plateau at temperatures of 10 to $20 \mathrm{~K}$. Again this is similar to what is found in amorphous solids and irradiated quartz. The plateau corresponds to the regime $\omega \tau_{\mathrm{m}}<<1$ as predicted by the tunneling model (see eq. 3). Also at these temperatures the attenuation is very small compared to the plateau in vitreous silica: a factor of about 100 is found, which is similar than for the lowest temperatures. Preliminary 
experiments at two other frequencies showed that the height of the plateau is proportional to the frequency, which is again in agreement with the tunneling model (see eq. 3 ).

At still higher temperatures the plateau is masked by other processes. It is clear from fig. 1 that the anharmonic three-phonon processes which are typical for the pure crystal start to become important around $25 \mathrm{~K}$ and mask the attenuation by the TS. In addition it is not to be ruled out that at these temperatures structural relaxation processes become important as it is the case for amorphous solids and irradiated quartz, where also only a shoulder is observed in the ultrasonic attenuation instead of a plateau. These processes are also due to two-state defects, however the transition from one level to the other occurs by thermal activation [1].

Comparing again with the results found for vitreous silica and with those for neutron-irradiated quartz, it is seen that the transition from the $\omega \tau_{\mathrm{m}} \gg 1$ regime to the $\omega \tau_{\mathrm{m}}<<1$ regime in neutron-irradiated Si takes place at higher temperatures than for vitreous silica or neutron-irradiated quartz. This means that the coupling between the phonons and the TS will be smaller. This is consistent with the fact that the network is more rigid in the case of $\mathrm{Si}$.

\section{CONCLUSIONS}

Ultrasonic attenuation measurements were carried out on two single-crystalline bulk Si samples, one of them remained in its pure crystalline form and the other one was partly disordered by irradiating it with a fastneutron dose of $1.7 \times 10^{21} \mathrm{n} / \mathrm{cm}^{2}$.

From the data obtained from ultrasonic attenuation measurements as a function of temperature, it can be concluded that due to neutron irradiation, a large additional effect in the ultrasonic attenuation is present: the unirradiated sample shows no changes in attenuation until $25 \mathrm{~K}$, while for the neutron-irradiated sample changes can already be detected at $1 \mathrm{~K}$.

It can also be noticed that the behavior of the ultrasonic attenuation as a function of temperature for neutron-irradiated Si shows a remarkable similarity with the predictions of the tunneling model for partly disordered materials: a $T^{3}$-dependence at low temperatures is followed by a temperature-independent part at higher temperatures. This is an indication for the fact that due to neutron-irradiation, TS are induced into the bulk single-crystalline $\mathrm{Si}$.

\section{Acknowledgements}

The authors are indebted to SCK-CEN for carrying out the irradiations. They are grateful to F.B.F.C. in Dessel (Belgium) for the use of the equipment for the mass density measurements and they are also thankful to the Belgian IIKW for financial support.

\section{References}

[1] Anderson P.W., Halperin B.I. and Varma C.M., Philos. Mag. 25 (1972) 1. Phillips W.A., J. Low Temp. Phys. 7 (1972) 351.

[2] See for instance Laermans C. and Keppens V., Phys. Rev. B 51 (1995) 8158.

[3] Graebner J.E., Golding B., Allen L.C., Knights J.C. and Biegelsen D.K, Phys. Rev. B 29 (1984) 3744.

[4] Graebner J.E. and Allen L.C., Phys. Rev. B 29 (1984) 5626.

[5] von Haumeder M., Strom U. and Hunklinger S., Phys. Rev. Lett. 44 (1980) 84.

[6] can be deduced from Doussineau P., Frénois C., Leisure R.G., Levelut A., and Prieur J.Y., J.Phys. (Paris) 41 (1980) 1193.

[7] Laermans C. and Coeck M., Czech. J. Phys. 46 (1996) Suppl. S4, 2225-2226.

[8] Hunklinger S., private communication Barttell U., $\mathrm{PhD}$ thesis, University of Heidelberg

[9] Jäckle J., Piché L., Arnold W. and Hunklinger S., J. Non-Cryst. Solids 20 (1976) 365. 